\title{
NoTE
}

\section{Up-Regulation of JAM-1 in AR42J Cells Treated with Activin A and Betacellulin and the Diabetic Regenerating Islets}

\author{
YUKAKO YOSHIKUMI, HIDEKI OHNO, JUNKO SUZUKI, MASASHI ISSHIKI*, YASUYUKI MORISHITA**, \\ HIROHIDE OHNISHI***, HIROSHI YASUDA ${ }^{\#}$, MASAO OMATA, TOSHIRO FUJITA* AND HIROSATO MASHIMA \\ Department of Gastroenterology, Graduate School of Medicine, University of Tokyo, Tokyo 113-8655, Japan \\ *Endocrinology and Nephrology, Graduate School of Medicine, University of Tokyo, Tokyo 113-8655, Japan \\ **Pathology, Graduate School of Medicine, University of Tokyo, Tokyo 113-8655, Japan \\ ***Division of Gastroenterology and Neurology, Akita University School of Medicine, Akita 010-8543, Japan \\ \#Division of Gastroenterology, Showa University Fujigaoka Hospital, Kanagawa 227-8501, Japan
}

\begin{abstract}
Pancreatic AR42J cells demonstrate the pluripotency in precursor cells of the gut endoderm and also provide an excellent model system to study the differentiation of the pancreas. Using the mRNA differential display technique, we identified junctional adhesion molecule-1 (JAM-1), a component of the tight junction, was highly up-regulated during the differentiation of AR42J cells, although junctions were not formed. The expression level of JAM-1 showed an upregulation in the mRNA level after 3 hours and in the protein level after 24 hours in [activin A + betacellulin]-treated AR42J cells. The expressions of its signaling molecules, PAR-3 and atypical PKC $\lambda$, also increased after the addition of activin A + betacellulin. When JAM-1 was over-expressed in [activin A + betacellulin]-treated AR42J cells, tagged-JAM-1 was observed in cytoplasm as vesicular structures and JAM-1 was colocalized with Rab3B and Rab13, members of the Rab family expressed at tight junctions. In streptozotocin-induced regenerating islets, the expression of JAM-1 was also up-regulated in the mRNA level and the protein level. JAM-1 might therefore play an important role in the differentiation of AR42J cells and the regeneration of pancreatic islets.
\end{abstract}

Key words: JAM-1, AR42J cells, Activin A, Betacellulin, Islet

(Endocrine Journal 55: 757-765, 2008)

BOTH endocrine and exocrine cells of the pancreas arise from epithelial cells in the pancreatic duct $[1,2]$. Recent genetic studies indicate that pancreatic development depends on an integrated network of distinct transcription factors operating at various levels. A mouse homeobox protein, called insulin promoter

Received January 17, 2008

Accepted Janurary 18, 2008

Correspondence to: Hirosato MASHIMA, M.D., Ph.D., Department of Gastroenterology, Graduate School of Medicine, University of Tokyo, 7-3-1 Hongo, Bunkyo-ku, Tokyo 113-8655, Japan Act, Activin A; BTC, Betacellulin; JAM, junctional adhesion molecule; TJ, tight junction; aPKC $\lambda$, atypical PKC $\lambda$; MDCK, Mardin-Darby canine kidney; STZ, streptozotocin; Dx, dexamethasone; GLP-1, glucagon-like peptide 1; AJ, adherens junction; $\mathrm{ZO}-1$, zona accludens-1; Ab, antibody; mAb, monoclonal antibody; pAb, polyclonal antibody; PBS, phosphate buffered saline; BHK, baby hamster kidney; PP, pancreatic polypeptide. factor-1 (IPF-1/PDX-1), is required for the development of the pancreas [3]. Reduced Notch signaling during embryogenesis leads to an increased expression of neurogenin3, which promotes the endocrine fate. Conversely, at normal levels of Notch signaling, cells express HES-1 and bHLH protein PTF1-p48 adopt the exocrine fate [4-6]. Islet-1, Beta-2/NeuroD, Pax4 and Pax6 are necessary for the development and generation of mature islet cells [4-6]. Embryonic stem cells have been shown to differentiate into insulin-secreting structures similar to pancreatic islets [7].

Rat AR42J cells are derived from a chemically induced pancreatic tumor and demonstrate the features of pluripotency of the common precursor cells of the gut endoderm [7]. When exposed to dexamethasone (Dx), they become more acinar-like cells [8] and when incubated with Dx for a long period of time, they synthesize albumin and take on the characteristics of 
hepatocytes [9]. As we have shown, when AR42J cells were treated with activin A (Act), a member of the TGF- $\beta$ superfamily, they extend neuron-like processes and express pancreatic polypeptide (PP) [10, 11]. When incubated with Act and betacellulin (BTC), a member of the EGF superfamily, or hepatocyte growth factor, these cells differentiate into insulinproducing cells [11, 12]. Moreover, when AR42J cells were incubated with glucagon-like peptide 1 (GLP-1), they differentiate into insulin- and/or glucagonproducing cells [13]. In this way, AR42J cells resemble the pluripotent cells in the developing gut endoderm and provide an excellent in vitro model system to study the differentiation of both endocrine and exocrine pancreatic cells. To clarify the molecular mechanisms of differentiation in the pancreas, we used the method of mRNA differential display and identified numerous genes that were up- or down-regulated during the differentiation of AR42J cells [14]. Among them, we have focused our attention on one gene, which was highly up-regulated when treated with Act + BTC. This gene was a rat orthologous protein to mouse JAM-1.

JAM-1 belongs to an immunoglobulin superfamily and it is one of the proteins that contribute to the formation of tight junctions (TJs) $[15,16]$. TJ is the most apical junction in epithelial and endothelial cells and is implicated in multiple functions such as the barrier, fence, and signaling functions [17-19]. Another type of cell to cell junctional structures is adherens junction (AJ). AJ is formed by clusters of transmembrane proteins belonging to the cadherin family linked intracellulary to catenines and the actin cytoskeleton [20]. Occludin [21, 22], claudins [23, 24], and JAMs [15, 25-27] are integral membrane proteins localized at TJs. Many cytosolic proteins have been reported to be associated with the cytoplasmic surface of TJs such as ZO-1 (zonula occludens-1), ZO-2, ZO-3, and several PDZ-containing proteins [19]. JAM-1 was shown to bind directly to ZO-1 [28] and PAR-3 [29-31], a mammalian homologue of the par-3 gene product, at their PDZ domains. PAR-3 forms a ternary complex with PAR- 6 and atypical PKC $\lambda$ (aPKC $\lambda$ ) and these three proteins play a critical role in the apico-basal polarization of mammalian epithelial cells [31].

The Rab family small $G$ proteins consist of more than 60 family members and participate in determining the specificity of vesicular transport pathways. Two family members, Rab3B and Rab13, localize to TJs in polarized epithelial cells and cytoplasmic vesicular structures in non-polarized fibroblasts $[32,33]$. Epithelial cells lose their intercellular junctions after a decrease in the extracellular calcium concentration and in T84 intestinal epithelial cells, the junctional proteins were shown to translocate from lateral cell membrane into cytoplasmic ring-like structures directly underneath the apically localized F-actin [34]. Rab3B and Rab13 were also shown to be the regulators of polarized transport of basolateral and $\mathrm{TJ}$ membrane proteins in fibroblastic baby hamster kidney (BHK) cells [35].

We wondered why the expression of JAM-1 was upregulated during the differentiation of AR42J cells, when [Act + BTC]-treated AR42J cells stopped proliferation, extended the neuron-like processes, and did not form junctions. JAM-1 was reported to be expressed considerably in the cells lacking TJs such as platelets and leukocytes [15]. In platelets, JAM-1 plays a role in the activation and aggregation and JAM-1 participates in the sequential steps of adhesion and subsequent transmigration during inflammatory recruitment of leukocytes [36]. In this study, to understand the role of JAM-1, we examined the change of the expression level of JAM-1 and its subcellular localization in AR42J cells, and compared the expression level between normal and diabetic regenerating islets.

\section{Materials \& Methods}

\section{Materials}

Recombinant human Act was provided by Dr. Y. Eto of the Central Research Laboratory, Ajinomoto Inc. (Kawasaki, Japan). Recombinant human BTC [37] was generously provided by Dr. M. Seno of Okayama University (Okayama, Japan). The antibodies (Abs) used in this study were anti-JAM-1 polyclonal antibody $(\mathrm{pAb})$ and anti-actin pAb from Santa Cruz Biotechnology, anti-ZO-1 pAb from Zymed Laboratories Inc., anti-insulin pAb from abcam, antiglucagon pAb from Progen Biotechnik GmbH, antimyc monoclonal antibody ( $\mathrm{mAb}$ ) from Invitrogen Life Technologies, and anti-FLAG pAb from Sigma-Aldrich. Anti-PAR3 and anti-aPKC $\lambda$ pAbs were kindly provided from Dr. S. Ohno of Yokohama Civil University (Yokohama, Japan) and anti-JAM-1 mAb was a generous gift from Dr. T. Kita of Kyoto University 
(Kyoto, Japan). Secondary antibodies were FITCconjugated anti-rabbit IgG and $\mathrm{Cy} 3$-conjugated antimouse IgG from Jackson Immuno Research Laboratories (West Grove, PA) and Alexa Fluor anti-rabbit IgG and anti-mouse IgG from BD Biosciences.

\section{Cell culture}

AR42J and Mardin-Darby canine kidney (MDCK) cells were cultured in DME medium supplemented with $10 \mathrm{mM}$ HEPES, $10 \%$ heat-inactivated fetal calf serum, $2 \mathrm{mM}$ L-glutamine, $100 \mathrm{U} / \mathrm{ml}$ penicillin, $100 \mathrm{mg} / \mathrm{ml}$ streptomycin, and $1 \mathrm{mM}$ sodium pyruvate. A pancreatic alpha cell line ( $\alpha \mathrm{TC} 1.6)$ and a beta cell line (Min6) were also cultured in DME medium as described previously [38]. $\alpha \mathrm{TC} 1.6$ cells were kindly provided by Dr. K. Hamaguchi (Oita University, Japan). To stain cells, cells were grown on non-coated cover slips.

\section{Western Blotting}

Protein extracts from AR42J cells and rat pancreatic islets were prepared for immunoblotting as described previously [39]. For Western blotting, the membranes were blocked by incubation for $1 \mathrm{~h}$ with Blocking Ace (Snow Brand Milk Products, Sapporo, Japan). After blocking, the membranes were incubated with first $\mathrm{Ab}$ for $1 \mathrm{~h}$ at room temperature, washed three times in phosphate buffered saline (PBS) containing $0.1 \%$ Tween 20 for $10 \mathrm{~min}$, and incubated for $30 \mathrm{~min}$ with horseradish peroxidase-conjugated goat anti-rabbit or anti-mouse whole immunoglobulin (Nordic, 1:500 in PBS, 0.1\% Tween 20). After washing, an enhanced chemiluminescence Western blotting detection reagent (Amersham Biosciences, Piscataway, NJ) was added, and the reaction was allowed to proceed according to manufacturer's recommendations. Exposure to X-ray film was $1-3 \mathrm{~min}$ at room temperature. To remove the probe, the membranes were incubated with the stripping buffer containing $62.5 \mathrm{mM}$ Tris/ $\mathrm{HCl}$ (pH6.7), 2\% SDS, and $0.1 \mathrm{M} 2$-mercaptoethanol for $30 \mathrm{~min}$ at $50^{\circ} \mathrm{C}$.

\section{Immunofluoresence staining}

Effectene Transfection Reagent (Qiagen) was used for transfection according to the manufacturer's instructions. Forty-eight hours after the transfection, the cells were fixed for $30 \mathrm{~min}$ in $3 \%$ paraformaldehyde in
PBS. Specimens were treated with $0.1 \%$ ( $\mathrm{vol} / \mathrm{vol})$ Triton X-100 in PBS for 5 min, and incubated sequentially with Blocking Ace (Snow Brand Milk Products, Sapporo, Tokyo, Japan), first Ab, and second Ab. The specimens were examined under a fluorescent microscope (Axiophoto; Carl Zeiss, Inc., Thornwood, NY).

\section{Construction of Expression Vectors}

To construct the tagged JAM-1 expression vector (JAM-1/myc-His), we amplified a fragment of JAM-1 (nucleotides 73-972) by PCR using the sense primer (5'-gaagcttgccaccatgggcaccgaggggaaagcc-3') and the antisense primer (5'-gctcgagcgcaccaggaatgacgaggtctg3'), followed by HindIII and XhoI digestion. The fragment was subcloned into the HindIII/XhoI site of the vector, pcDNA3.1/myc-His (Invitrogen, Carlasbad, $\mathrm{CA}$ ), and was verified by sequencing. To construct FLAG-tagged Rab3B and Rab13 expression vector (Rab3B/FLAG, Rab13/FLAG), we amplified fragments of rat Rab 3B (nucleotide 4-660, GenBank Accession Number NM_031091) and human Rab13 (nucleotide 98-706, GenBank Accession Number NM_002870) using the sense primer (5'-caagcttgcctc agtaactgatggtaac-3', 5'-caagcttgccaaagcctacgaccacctc$\left.3^{\prime}\right)$ and the antisense primer (5'-gaattcgcetagcaagagc agttctgctgg-3', 5'-gaattcgctcagcccagggagcacttgttgg-3') respectively. After digestion by HindIII and EcoRI, the fragments were subcloned into the HindIII/EcoRI site of the vector, pFLAG-CMV-2 expression vector (Sigma, Missouri, USA), and then were verified by sequencing.

\section{Streptozotocin (STZ) treatment and measurement of plasma glucose levels}

Male Wistar rats weighing approximately $300-325 \mathrm{~g}$

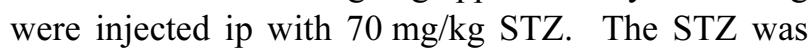
dissolved in $0.2 \mathrm{ml}$ of the citrate/phosphate buffer (31 $\mathrm{mM}$ citrate, $39 \mathrm{mM} \mathrm{NaPi}$; $\mathrm{pH} 4.0-4.5$ ). Control animals received injections of $0.2 \mathrm{ml}$ of the citrate/phosphate buffer alone and then were followed for the same periods of time as the STZ-treated animals. The plasma glucose levels were determined and any animals displaying plasma glucose levels greater than $400 \mathrm{mg} /$ $\mathrm{dl}$ were considered to be diabetic. All rat experiments were conducted in accordance with the National Institutes of Health Guidelines for the Care and Use of Laboratory Animals and were approved by the Univer- 
sity of Tokyo Institutional Animal Care and Use Committee.

\section{Analysis of mRNA by Reverse Transcription-PCR}

The rat pancreatic islets were prepared as described elsewhere [40]. To eliminate the contamination of acinar cells, we picked up islets from the buffer and repeated the selection from the fresh buffer. Total RNA was extracted from pancreatic islets using Trizol Reagent (Invitrogen, Carlsbad, CA). For semi-quantitative RT-PCR, first-stranded cDNA was synthesized by using Superscript ${ }^{\mathrm{TM}}$ First-stranded Synthesis System for RT-PCR (Invitrogen, Carlsbad, CA) according to the manufacturer's instructions. Oligonucleotide primers used were 5'-gacctgctcagaacacgac-3' (nucleotides 522-540) and 5'-tgggctggctgtaaatgacc-3' (nucleotides 925-906) for JAM-1 (404 bp PCR product) and 5'-tgagagggaaatcgtgcgtg-3' (nucleotides 612-631) and 5'-gatccacatctgctggaaggtg-3' (nucleotides 10711051 ) for $\beta$-actin ( $460 \mathrm{bp}$ PCR product, Accession No. V01217). The number of PCR cycles for semi-quantitative RT-PCR were thirty for JAM-1, and twenty-five for $\beta$-actin.

\section{Results}

\section{Molecular cloning of rat JAM-1}

Using the mRNA differential display technique, we identified several novel genes, which were highly upregulated during the differentiation of the AR42J cells. Among them we focused our attention on one gene (AB15) and the size of mRNA was estimated to be about $2.0 \mathrm{~kb}$ according to the result of a Northern blot analysis [14]. From the rat islet cDNA library, we obtained a novel 1895-bp cDNA, which comprises an open reading frame of $900 \mathrm{bp}, 72-\mathrm{bp} 5$ '-UTR, and a 923-bp 3'-UTR with a poly(A) tail and deposited to a GenBank database (GenBank Accession No. AF276998). There might be some additional regions of the 5'-UTR to be cloned; however, there was an inframe stop codon in the 5'-UTR of the sequence. The open reading frame encoded a predicted polypeptide of 300 amino acids with a calculated molecular mass of $32.4 \mathrm{kDa}$. With the Blast search, this gene had $91.0 \%$ identity and $96.7 \%$ similarity to mouse JAM-1 ([15], GenBank Accession No. O88792) and it was consid-

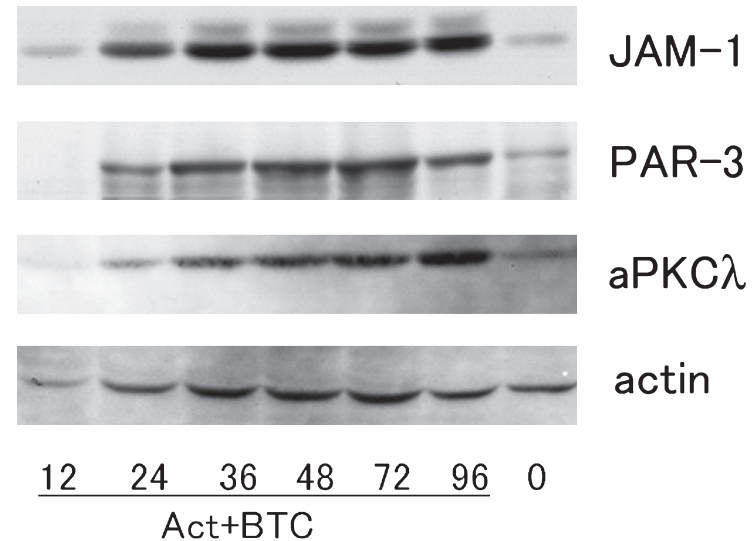

Fig. 1. Time Course of the Expression of JAM-1, PAR3 and aPKC $\lambda$ in $[$ Act + BTC]-treated AR42J cells. Total cell lysate was prepared from [Act + BTC]-treated AR42J cells at $0,12,24,36,48,72,96 \mathrm{hrs}$ and Western blot analyses were performed. The experiments were repeated three times and the representative figures were shown.

ered to be rat JAM-1.

\section{Changes of expression levels of JAM-1 and its signaling molecules}

The mRNA level of JAM-1 in AR42J cells rapidly increased in response to Act + BTC within 3 hrs as we showed previously [14]. First of all, we studied the changes in the protein level of JAM-1 and its signaling molecules; PAR-3 and aPKC $\lambda$. As shown in Fig. 1, the protein level of JAM-1 was up-regulated at $24 \mathrm{hrs}$ after the addition of Act + BTC and the expressions of PAR-3 and aPKC $\lambda$ were also observed to increase after a slight decrease at $12 \mathrm{hrs}$.

\section{Expression of JAM-1 in AR42J cells}

JAM-1 is ubiquitously expressed in epithelial and endothelial cells. Anti-JAM-1 mAb, used in this study, was kindly provided by Dr. T. Kita (Kyoto University, Japan) and this mAb specifically stained JAM1 in MDCK cells and the staining was identical to that of ZO-1 (Fig. 2A). When JAM-1 was over-expressed in MDCK cells using the expression vector, JAM-1/ myc-His, and was stained with anti-myc mAb, cell-tocell was also stained in a transfected cell (Fig. 2B). JAM-1 was up-regulated in both the mRNA level and the protein level when AR42J cells differentiate in response to Act + BTC. However, when we stained 


\section{A. MDCK}

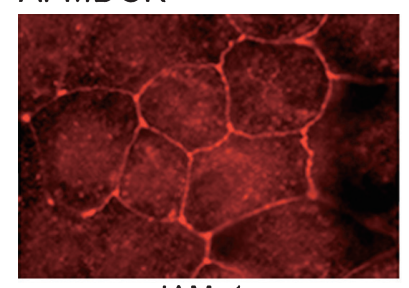

$\mathrm{JAM}-1$

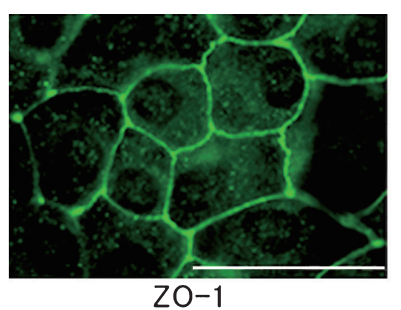

B. MDCK $/ /$ JAM-1/myc-His

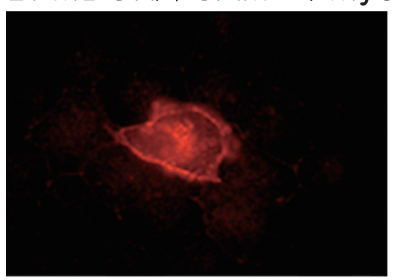

myc

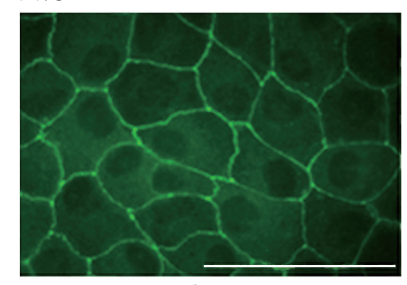

$\mathrm{ZO}-1$

C. naive AR42J//JAM-1/myc-His
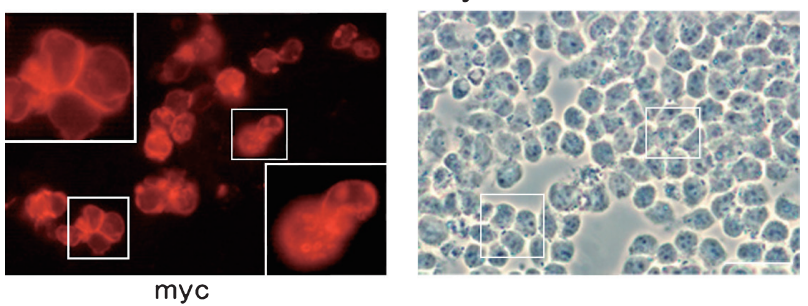

D. $[$ Act + BTC $]-A R 42 J / / J A M-1 / m y c-H i s$
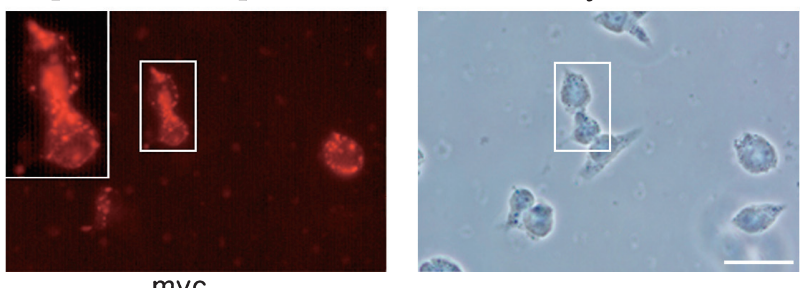

myc

Fig. 2. Expression of JAM-1 in MDCK and AR42J cells. Naïve MDCK (A) and JAM-1 over-expressed MDCK cells (B) were stained with anti-JAM-1, anti-myc, and anti-ZO-1 Abs. AR42J cells were transfected with JAM-1/myc-His expression vector, incubated without (C) or with (D) Act + BTC, and stained with anti-myc $\mathrm{Ab}$. The panels on the right showed phase-contrast images. Bars, $50 \mu \mathrm{m}$.

naïve AR42J cells and [Act + BTC]-treated AR42J cells at 24, 72, and $168 \mathrm{hrs}$, we could not detect any signals of JAM-1 (Data not shown). Therefore, JAM-1 was over-expressed in AR42J cells with or without Act + BTC to study the localization of JAM-1. In naïve AR42J cells, JAM-1 was observed not only in cell-to-cell contact but also in cytoplasm as vesicular structures (Fig. 2C). When incubated with Act + BTC, AR42J cells stopped proliferation, extended neuronlike processes, and did not form junctions between the surrounding cells. JAM-1 was detected in cytoplasm as vesicular structures in JAM-1 over-expressed AR42J cells (Fig. 2D).

Colocalization of JAM-1 with Rab3B and Rab13 in AR42J cells

Of the Rab family members, Rab3B and Rab13 preferentially localized to TJs in polarized epithelial cells $[32,33]$. Using a model of calcium depletion, the endocytosis of junctional proteins was shown to be mediated in a clathrin-dependent process [34]. We then examined whether the staining of JAM-1 in the cytoplasm was colocalized with Rab3B and/or Rab13. We co-transfected JAM-1/myc-His and Rab3B/FLAG or Rab13/FLAG expression vectors into AR42J cells and stained the cells with anti-myc and anti-FLAG Abs. JAM-1 and Rab3B (Fig. 3A) and JAM-1 and Rab13 (Fig. 3B) colocalized in the cytoplasm as vesicular structures.

\section{Expression of JAM-1 in STZ-induced diabetic islet}

In the next set of experiments, we injected STZ, a specific $\beta$-cell toxin, to rats intrapenitoneally resulting in the stimulation of islet regeneration and compared the expression level of JAM-1. JAM-1 was up-regulated in the STZ-induced diabetic islets both on the mRNA level (Fig. 4A) and on the protein level (Fig. 4B). The expression of aPKC $\lambda$ also increased in the diabetic islets.

\section{Discussion}

In the present study, we identified a novel cDNA whose expression level was highly up-regulated when AR42J cells differentiate in response to Act + BTC [14] and it turned out to be a rat orthologous protein to mouse JAM-1. JAM-1 belongs to an immunoglobulin superfamily and characterized by two Ig-like folds (One $\mathrm{V}_{\mathrm{H}^{-}}$-type and one $\mathrm{C}_{2}$-type) in the extracellular domain, a single transmembrane domain, and a short intracellular tail that features a classical type II PDZ binding motif [15]. TJ is the most apical junction in epithelial and endothelial cells and it is implicated in 
A
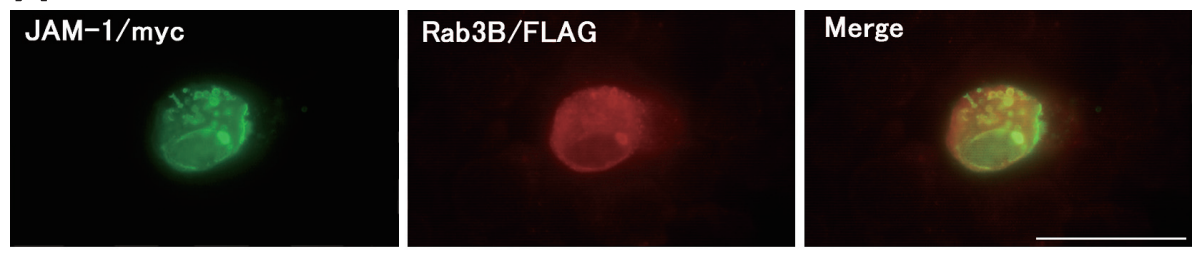

B
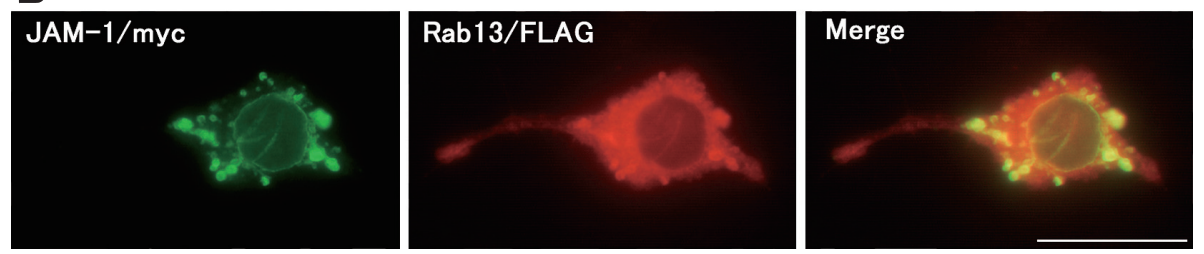

Fig. 3. Colocalization of JAM-1 with Rab3B and Rab13 in AR42J cells. AR42J cells were cotransfected with JAM-1/myc-His and Rab3B/FLAG (A) or Rab13/FLAG (B) expression vectors. The cells were stained with anti-myc mAb and anti-FLAG pAb and then were examined. Bars, $20 \mu \mathrm{m}$.
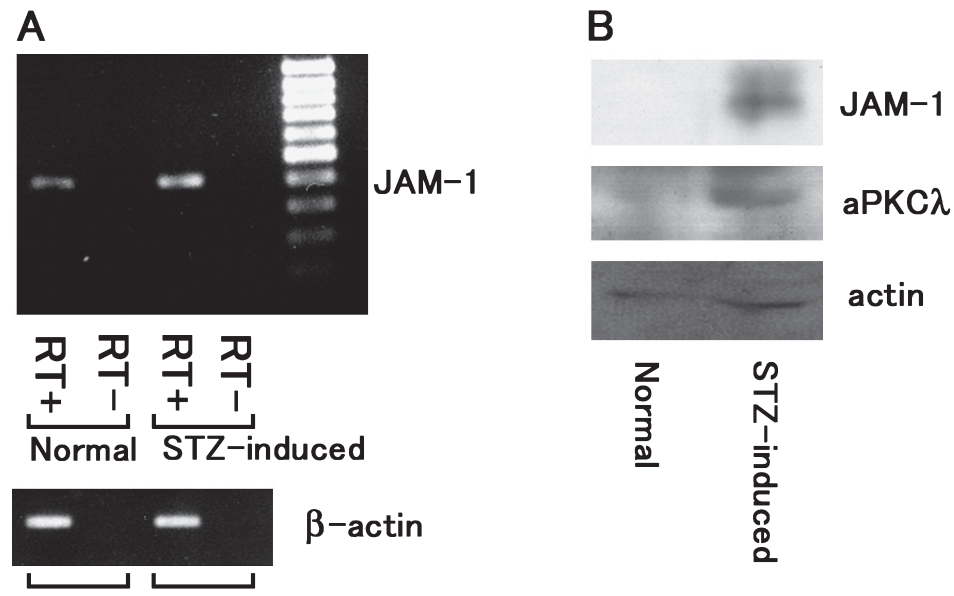

Fig. 4. Expressions of JAM-1 in STZ-induced Diabetic Islet. A. mRNA of JAM-1 was compared by RT-PCR in normal and STZinduced rat $(6 \mathrm{~W})$ pancreatic islets. Samples with $(\mathrm{RT}+)$ or without $(\mathrm{RT}-)$ reverse transcriptase treatment were loaded. The right end lane is a 100-bp ladder size marker. mRNA of $\beta$-actin was also compared (lower panel). B. The protein level of JAM-1 was compared by a Western blot analysis in normal and STZ-induced rat $(6 \mathrm{~W})$ pancreatic islets. The blot was reprobed with anti-aPKC $\lambda$ and anti-actin pAbs.

multiple functions such as barrier, fence, and signaling functions [17-19]. JAM-1 binds directly to the PDZ domain of PAR-3 with its PDZ binding motif [29-31] and PAR-3 forms a ternary complex with PAR-6 and aPKC $\lambda$ [31]. The expression levels of JAM-1, PAR-3, and aPKC $\lambda$ increased in response to Act + BTC within $36 \mathrm{hrs}$ in protein level (Fig. 1), thus suggesting that these molecules might play an important role at an early phase in the differentiation of AR42J cells.

JAM forms a family from JAM-1 to JAM-4. JAM-2 [25] and JAM-3 [26] are largely confined to endothelial cells. JAM-4 has been described as an interacting protein with TJ protein MAGI-1 (MAGUK with inverted domain structure) [27]. Recently JAM-C (JAM-3 in H. sapiens and JAM-2 in M. musculus) was reported to be essential for the polarization of round spermatids and critically required for the differentiation of round spermatids into spermatozoa in mice [41]. It was also reported that JAM-1 contributed to the differentiation of the trophectoderm in the mouse embryo and JAM-1 recruitment to cell contact sites occurred earlier than any other TJ protein and E-cadherin [42].

AR42J cells have the features of common precursor 
cells of the pancreas [10, 43]. The expression of PDX-1 is the earliest and most specific endoderm marker of the developing pancreas [3] and PDX-1 is expressed abundantly in naïve AR42J cells [44]. In the mouse embryo, JAM-1-encoding mRNA is expressed early from the embryonic genome and is detectable as protein from the eight-cell stage [42]. JAM-1 may exert an important function in the early stage of the differentiation of common endocrine precursor cells.

We could not detect the staining of JAM-1 in naïve and [Act + BTC]-treated AR42J cells, though the expression of JAM-1 was up-regulated in [Act + BTC]treated cells (Data not shown), and this finding is difficult to interpret. Ozaki, et al. used the same mAb and reported that the combined treatment of proinflammatory cytokines, TNF- $\alpha+$ IFN- $\gamma$, caused disappearance of JAM-1 from intercellular junctions in human umbilical vein endothelial cells (HUVECs) [45]. Flow cytometry, cell ELISA, and subcellular fractionation analyses demonstrated that the amount of JAM-1 of cell surface was not reduced, thus suggesting that JAM-1 was redistributed to the cell surface from TJs. To detect JAM-1 with this $\mathrm{mAb}$, the concentrated expression of JAM-1 might therefore be needed and distributed JAM-1 could not be detected probably due to conformational changes. The X-ray structure of JAM-1 suggests a homophilic adhesion model in which U-shaped JAM dimmers stick out almost perpendicular to the cell surface. Contact is established between the first variable type amino terminal loops that lie almost parallel to the cell surface [46]. It was reported that in Chinese hamster ovary cells, exogenously expressed JAM-1 was localized to newly formed cell-cell borders and in mixed cultures with control transfectants, JAM-1 remained diffuse [15].

It was recently reported that a pool of occludin was continuously endocytosed and recycled back to the cell surface in fibroblastic and epithelial cells and Rab13 specifically mediated the continuous endocytic recycling of occludin [47]. The internalization of TJs appears to be a common mechanism to rapidly downregulate cell-cell adhesion and allow remodeling of intercellular junctions. Internalization is also induced by various pathophisiological stimuli including bacterial products, proinflammatory cytokines, and oxidative stress [36]. Epithelial cells lose intercellular junctions after decrease in extracellular calcium concentration to the micromolar range and these events are associated with the rapid internalization of junctional proteins in a clathrin-mediated process [36]. Rab3B and Rab13 localize to tight junctions in polarized epithelial cells and cytoplasmic vesicular structures in non-polarized fibroblasts [32, 33]. Rab3B and Rab13 are shown to regulate the polarized transport of the basolateral and TJ membrane proteins in BHK cells [35]. We then examined whether JAM-1 was colocalized with Rab3B and/or Rab13 in [Act + BTC]treated AR42J cells. As shown in Fig. 3, Rab3B and Rab13 were colocalized with JAM-1 in the cytoplasmic vesicular structures, thus suggesting that JAM-1 in the cytoplasm was regulated by these molecules.

We next examined the expression of JAM-1 in STZinduced regenerating islets. STZ destroys functional $\beta$-cells and induces diabetes in these animals, thus resulting in the stimulation of the regeneration of the islets $[48,49]$. JAM-1 was up-regulated in STZ-induced regenerating islet at $6 \mathrm{~W}$ both at the mRNA level and the protein level, suggesting that JAM-1 plays an important role in the regeneration of pancreatic islets. When JAM-1 was over-expressed in a pancreatic beta cell line (Min6) and an alpha cell line ( $\alpha \mathrm{TC} 1.6)$, the immunoreactivity to insulin or glucagon was not altered in the transfected cells (Data not shown).

The function of JAM-1 in AR42J cells and pancreatic islet is still unclear; nevertheless the increased expression of JAM-1 in [Act + BTC]-treated AR42J cells and STZ-induced regenerating islets suggested that JAM-1 might play an important role in the differentiation of AR42J cells and the regeneration of the pancreatic islets. Further studies, including the intense observations of JAM-1 in islets of the fetal stage and a knockdown experiment of JAM-1 in AR42J cells, are therefore needed to confirm our speculations.

\section{Acknowledgements}

We thank Dr. M. Hayashi for technical assistance in the preparation of the pancreatic islets. Grant sponsor: A grant-in-Aid for Scientific Research from the Ministry of Education Science, Technology, Sports and Culture (to H. M., No. 17590616). 


\section{References}

1. Teitelman G, Lee JK (1987) Cell lineage analysis of pancreatic islet development: glucagon and insulin cells arise from catecholaminergic precursors present in the pancreatic duct. Dev Biol 121: 454-466.

2. Pictet RL, Clark WR, Williams RH, Rutter WJ (1972) An ultrastructural analysis of the developing embryonic pancreas. Dev Biol 29: 436-467.

3. Jonsson J, Carlsson L, Edlund T, Edlund H (1994) Insulin-promoter-factor 1 is required for pancreas development in mice. Nature 371: 606-609.

4. Apelqvist A, Li H, Sommer L, Beatus P, Anderson DJ, Honjo T, Hrabe de Angelis M, Lendahl U, Edlund H (1999) Notch signalling controls pancreatic cell differentiation. Nature 400: 877-881.

5. Gu G, Dubauskaite J, Melton DA (2002) Direct evidence for the pancreatic lineage: NGN3+ cells are islet progenitors and are distinct from duct progenitors. Development 129: 2447-2457.

6. Krapp A, Knofler M, Ledermann B, Burki K, Berney C, Zoerkler N, Hagenbuchle O, Wellauer PK (1998) The bHLH protein PTF1-p48 is essential for the formation of the exocrine and the correct spatial organization of the endocrine pancreas. Genes Dev 12: 3752-3763.

7. Lumelsky N, Blondel O, Laeng P, Velasco I, Ravin R, McKay R (2001) Differentiation of embryonic stem cells to insulin-secreting structures similar to pancreatic islets. Science 292: 1389-1394.

8. Logsdon CD, Moessner J, Williams JA, Goldfine ID (1985) Glucocorticoids increase amylase mRNA levels, secretory organelles, and secretion in pancreatic acinar AR42J cells. J Cell Biol 100: 1200-1208.

9. Shen CN, Slack JM, Tosh D (2000) Molecular basis of transdifferentiation of pancreas to liver. Nat Cell Biol 2: 879-887.

10. Ohnishi H, Ohgushi N, Tanaka S, Mogami H, Nobusawa R, Mashima H, Furukawa M, Mine T, Shimada O, Ishikawa $\mathrm{H}$, et al. (1995) Conversion of amylasesecreting rat pancreatic AR42J cells to neuronlike cells by activin A. J Clin Invest 95: 2304-2314.

11. Mashima H, Ohnishi $H$, Wakabayashi $K$, Mine $T$, Miyagawa J, Hanafusa T, Seno M, Yamada H, Kojima I (1996) Betacellulin and activin A coordinately convert amylase-secreting pancreatic AR42J cells into insulinsecreting cells. J Clin Invest 97: 1647-1654.

12. Mashima H, Shibata H, Mine T, Kojima I (1996) Formation of insulin-producing cells from pancreatic acinar AR42J cells by hepatocyte growth factor. Endocrinology 137: 3969-3976.

13. Zhou J, Wang X, Pineyro MA, Egan JM (1999) Glucagon-like peptide 1 and exendin-4 convert pancreatic AR42J cells into glucagon- and insulin-producing cells. Diabetes 48: 2358-2366.

14. Mashima H, Yamada S, Tajima T, Seno M, Yamada H,
Takeda J, Kojima I (1999) Genes expressed during the differentiation of pancreatic AR42J cells into insulinsecreting cells. Diabetes 48: 304-309.

15. Martin-Padura I, Lostaglio S, Schneemann M, Williams L, Romano M, Fruscella P, Panzeri C, Stoppacciaro A, Ruco L, Villa A, Simmons D, Dejana E (1998) Junctional adhesion molecule, a novel member of the immunoglobulin superfamily that distributes at intercellular junctions and modulates monocyte transmigration. J Cell Biol 142: 117-127.

16. Bazzoni G, Martinez-Estrada OM, Orsenigo F, Cordenonsi M, Citi S, Dejana E (2000) Interaction of junctional adhesion molecule with the tight junction components ZO-1, cingulin, and occludin. J Biol Chem 275: 20520-20526.

17. Schneeberger EE, Lynch RD (1992) Structure, function, and regulation of cellular tight junctions. $\mathrm{Am} \mathrm{J}$ Physiol 262: L647-L661.

18. Liu Y, Nusrat A, Schnell FJ, Reaves TA, Walsh S, Pochet M, Parkos CA (2000) Human junction adhesion molecule regulates tight junction resealing in epithelia. J Cell Sci 113 (Pt13): 2363-2374.

19. Tsukita S, Furuse M, Itoh M (2001) Multifunctional strands in tight junctions. Nat Rev Mol Cell Biol 2: 285-293.

20. Gumbiner BM (1996) Cell adhesion: the molecular basis of tissue architecture and morphogenesis. Cell 84: 345-357.

21. Furuse M, Fujita K, Hiiragi T, Fujimoto K, Tsukita S (1998) Claudin-1 and -2: novel integral membrane proteins localizing at tight junctions with no sequence similarity to occludin. J Cell Biol 141: 1539-1550.

22. Furuse M, Sasaki H, Fujimoto K, Tsukita S (1998) A single gene product, claudin-1 or -2 , reconstitutes tight junction strands and recruits occludin in fibroblasts. $J$ Cell Biol 143: 391-401.

23. Tsukita S, Furuse M (1999) Occludin and claudins in tight-junction strands: leading or supporting players? Trends Cell Biol 9: 268-273.

24. Tsukita S, Furuse M, Itoh M (1999) Structural and signalling molecules come together at tight junctions. Curr Opin Cell Biol 11: 628-633.

25. Palmeri D, van Zante A, Huang CC, Hemmerich S, Rosen SD (2000) Vascular endothelial junction-associated molecule, a novel member of the immunoglobulin superfamily, is localized to intercellular boundaries of endothelial cells. J Biol Chem 275: 19139-19145.

26. Arrate MP, Rodriguez JM, Tran TM, Brock TA, Cunningham SA (2001) Cloning of human junctional adhesion molecule 3 (JAM3) and its identification as the JAM2 counter-receptor. J Biol Chem 276: 45826 45832.

27. Hirabayashi S, Tajima M, Yao I, Nishimura W, Mori 
H, Hata Y (2003) JAM4, a junctional cell adhesion molecule interacting with a tight junction protein, MAGI-1. Mol Cell Biol 23: 4267-4282.

28. Ebnet K, Schulz CU, Meyer Zu Brickwedde MK, Pendl GG, Vestweber D (2000) Junctional adhesion molecule interacts with the PDZ domain-containing proteins AF-6 and ZO-1. J Biol Chem 275: 2797927988.

29. Itoh M, Sasaki H, Furuse M, Ozaki H, Kita T, Tsukita S (2001) Junctional adhesion molecule (JAM) binds to PAR-3: a possible mechanism for the recruitment of PAR-3 to tight junctions. J Cell Biol 154: 491-497.

30. Ebnet K, Suzuki A, Horikoshi Y, Hirose T, Meyer Zu Brickwedde MK, Ohno S, Vestweber D (2001) The cell polarity protein ASIP/PAR-3 directly associates with junctional adhesion molecule (JAM). Embo J 20: 3738-3748.

31. Ohno S (2001) Intercellular junctions and cellular polarity: the PAR-aPKC complex, a conserved core cassette playing fundamental roles in cell polarity. Curr Opin Cell Biol 13: 641-648.

32. Zahraoui A, Joberty G, Arpin M, Fontaine JJ, Hellio R, Tavitian A, Louvard D (1994) A small rab GTPase is distributed in cytoplasmic vesicles in non polarized cells but colocalizes with the tight junction marker ZO-1 in polarized epithelial cells. J Cell Biol 124: 101115.

33. Weber E, Berta G, Tousson A, St John P, Green MW, Gopalokrishnan U, Jilling T, Sorscher EJ, Elton TS, Abrahamson DR, et al. (1994) Expression and polarized targeting of a rab3 isoform in epithelial cells. $J$ Cell Biol 125: 583-594.

34. Ivanov AI, Nusrat A, Parkos CA (2004) Endocytosis of epithelial apical junctional proteins by a clathrinmediated pathway into a unique storage compartment. Mol Biol Cell 15: 176-188.

35. Yamamoto Y, Nishimura N, Morimoto S, Kitamura H, Manabe S, Kanayama HO, Kagawa S, Sasaki T (2003) Distinct roles of Rab3B and Rab13 in the polarized transport of apical, basolateral, and tight junctional membrane proteins to the plasma membrane. Biochem Biophys Res Commun 308: 270-275.

36. Ostermann G, Weber KS, Zernecke A, Schroder A, Weber C (2002) JAM-1 is a ligand of the beta(2) integrin LFA-1 involved in transendothelial migration of leukocytes. Nat Immunol 3: 151-158.

37. Seno M, Tada H, Kosaka M, Sasada R, Igarashi K, Shing Y, Folkman J, Ueda M, Yamada H (1996) Human betacellulin, a member of the EGF family dominantly expressed in pancreas and small intestine, is fully active in a monomeric form. Growth Factors 13: $181-191$.

38. Hirayama I, Tamemoto H, Yokota H, Kubo SK, Wang
J, Kuwano H, Nagamachi Y, Takeuchi T, Izumi T (1999) Insulin receptor-related receptor is expressed in pancreatic beta-cells and stimulates tyrosine phosphorylation of insulin receptor substrate-1 and -2 . Diabetes 48: $1237-1244$.

39. Kanzaki M, Zhang YQ, Mashima H, Li L, Shibata H, Kojima I (1999) Translocation of a calcium-permeable cation channel induced by insulin-like growth factor-I. Nat Cell Biol 1: 165-170.

40. Lacy PE, Kostianovsky M (1967) Method for the isolation of intact islets of Langerhans from the rat pancreas. Diabetes 16: 35-39.

41. Gliki G, Ebnet K, Aurrand-Lions M, Imhof BA, Adams RH (2004) Spermatid differentiation requires the assembly of a cell polarity complex downstream of junctional adhesion molecule-C. Nature 431: 320-324.

42. Thomas FC, Sheth B, Eckert JJ, Bazzoni G, Dejana E, Fleming TP (2004) Contribution of JAM-1 to epithelial differentiation and tight-junction biogenesis in the mouse preimplantation embryo. J Cell Sci 117: 55995608.

43. Rosewicz S, Vogt D, Harth N, Grund C, Franke WW, Ruppert S, Schweitzer E, Riecken EO, Wiedenmann B (1992) An amphicrine pancreatic cell line: AR42J cells combine exocrine and neuroendocrine properties. Eur $J$ Cell Biol 59: 80-91.

44. Zhang YQ, Mashima H, Kojima I (2001) Changes in the expression of transcription factors in pancreatic AR42J cells during differentiation into insulin-producing cells. Diabetes 50 Suppl 1: S10-S14.

45. Ozaki H, Ishii K, Horiuchi H, Arai H, Kawamoto T, Okawa K, Iwamatsu A, Kita T (1999) Cutting edge: combined treatment of TNF-alpha and IFN-gamma causes redistribution of junctional adhesion molecule in human endothelial cells. J Immunol 163: 553-557.

46. Kostrewa D, Brockhaus M, D'Arcy A, Dale GE, Nelboeck P, Schmid G, Mueller F, Bazzoni G, Dejana E, Bartfai T, Winkler FK, Hennig M (2001) X-ray structure of junctional adhesion molecule: structural basis for homophilic adhesion via a novel dimerization motif. Embo J 20: 4391-4398.

47. Morimoto S, Nishimura $\mathrm{N}$, Terai $\mathrm{T}$, Manabe $\mathrm{S}$, Yamamoto Y, Shinahara W, Miyake H, Tashiro S, Shimada M, Sasaki T (2005) Rab13 mediates the continuous endocytic recycling of occludin to the cell surface. J Biol Chem 280: 2220-2228.

48. Like AA, Rossini AA (1976) Streptozotocin-induced pancreatic insulitis: new model of diabetes mellitus. Science 193: 415-417.

49. Fernandes A, King LC, Guz Y, Stein R, Wright CV, Teitelman G (1997) Differentiation of new insulinproducing cells is induced by injury in adult pancreatic islets. Endocrinology 138: 1750-1762. 